\title{
Tratamento cirúrgico de fratura bilateral de mandíbula em paciente pediátrico:
}

\section{Relato de caso}

\author{
Surgical treatment of bilateral mandible fracture in a pediatric patient: Case report \\ Tratamiento quirúrgico de la fractura bilateral de mandíbula en un paciente pediátrico: Reporte
}

de caso

Recebido: 02/05/2021 | Revisado: 10/05/2021 | Aceito: 11/05/2021 | Publicado: 28/05/2021

\author{
Mirlany Mendes Maciel Oliveira \\ ORCID: https://orcid.org/0000-0002-4744-8094 \\ Universidade Federal de Uberlândia, Brasil \\ E-mail: mirlany.mendess@gmail.com \\ Lívia Bonjardim Lima \\ ORCID: https://orcid.org/0000-0002-9924-4077 \\ Universidade Federal de Uberlândia, Brasil \\ E-mail: liviabonjardim@hotmail.com \\ Verônica Martins Salge \\ ORCID: https://orcid.org /0000-0002-5650-3905 \\ Universidade Federal de Uberlândia, Brasil \\ E-mail: veronicamsalge@gmail.com \\ Daniela Meneses Santos \\ ORCID: https://orcid.org/0000-0002-8898-9508 \\ Universidade Federal de Uberlândia, Brasil \\ E-mail: danyymeneses@yahoo.com.br \\ Cláudia Jordão Silva \\ ORCID: https://orcid.org/0000-0002-7114-4859 \\ Universidade Federal de Uberlândia, Brasil \\ E-mail: cjordao1@gmail.com \\ Luiz Fernando Barbosa de Paulo \\ ORCID: https://orcid.org /0000-0003-1146-0777 \\ Universidade Federal de Uberlândia, Brasil \\ E-mail: luizfbpaulo@gmail.com
}

\begin{abstract}
Resumo
As fraturas de face em crianças exibem características únicas e diferenciáveis, sendo o manejo individualizado desde o diagnóstico, passando pelo tratamento, até a proservação do caso. Tais fraturas podem desencadear consequências significativas, como deformidades e comprometimento facial, possuindo a diferenciação com a população adulta especialmente quanto a quesitos anatômicos e sociais. Os principais objetivos no tratamento de fraturas de face em pacientes pediátricos são restauração da funcionalidade e proporcionar união óssea preservando o potencial de crescimento facial. Quando possível uma abordagem não cirúrgica deve ser indicada, porém em casos que necessitem de redução cirúrgica e fixação interna este tratamento deve ser realizado utilizando técnica cirúrgica menos traumática. O acompanhamento a longo prazo é recomendado para adequada avaliação da funcionalidade e estética final alcançados. O objetivo deste trabalho é apresentar o caso clínico de um paciente pediátrico que evoluiu com fratura bilateral de mandíbula após acidente automobilístico, tradado de forma cirúrgica.
\end{abstract}

Palavras-chave: Infância; Fratura; Oclusão dentária; Trauma.

\begin{abstract}
Fractures of the face in children exhibit unique and differentiable characteristics, with individualized management from diagnosis, through treatment, to the preservation of the case. Such fractures can trigger significant consequences, such as deformities and facial impairment, having differentiation with the adult population, especially regarding anatomical and social issues. The main objectives in the treatment of facial fractures in pediatric patients are to restore functionality and provide bone union while preserving the potential for facial growth. When possible, a non-surgical approach should be indicated, but in cases that require surgical reduction and internal fixation, this treatment should be performed using less traumatic surgical technique. Long-term follow-up is recommended for an adequate assessment of the functionality and final aesthetics achieved. The objective of this work is to present the clinical case of a pediatric patient who developed a bilateral fracture of the jaw after an automobile accident, translated into a surgical form.
\end{abstract}

Keywords: Childhood; Fracture; Dental occlusion; Trauma. 


\begin{abstract}
Resumen
Las fracturas de la cara en niños exhiben características únicas y diferenciables, con manejo individualizado desde el diagnóstico, pasando por el tratamiento, hasta la preservación del caso. Tales fracturas pueden desencadenar consecuencias importantes, como deformidades y deterioro facial, teniendo diferenciación con la población adulta, especialmente en lo que se refiere a aspectos anatómicos y sociales. Los principales objetivos en el tratamiento de las fracturas faciales en pacientes pediátricos son restaurar la funcionalidad y proporcionar unión ósea preservando el potencial de crecimiento facial. Cuando sea posible, debe indicarse un abordaje no quirúrgico, pero en los casos que requieran reducción quirúrgica y fijación interna, este tratamiento debe realizarse utilizando una técnica quirúrgica menos traumática. Se recomienda un seguimiento a largo plazo para una adecuada valoración de la funcionalidad y estética final conseguida. El objetivo de este trabajo es presentar el caso clínico de un paciente pediátrico que desarrolló una fractura bilateral de mandíbula tras un accidente automovilístico, traducida a una forma quirúrgica.
\end{abstract}

Palabras clave: Infancia; Fractura; Oclusión dental; Trauma.

\title{
1. Introdução
}

As fraturas faciais em pacientes pediátricos podem desencadear morbidades significativas, como deformidades e comprometimento facial e diferem das populações adultas em virtude de fatores anatômicos e sociais (Gebran et al., 2019; Moffitt et al., 2019).

Mesmo em casos de traumas faciais o protocolo de Suporte Avançado de Vida em Trauma deve ser seguido rigorosamente, devendo ser avaliado, via aérea pérvia, respiração e circulação, bem como realizada avaliação secundária. Uma das principais observações iniciais em casos de fraturas de mandíbula em pacientes pediátricos é a permeabilidade das vias aéreas. Sinais clínicos que incluem edema, equimose, lacerações, má-oclusão dentária e trismo podem ser sugestivos de fraturas de mandíbula. O exame bimanual pode evidenciar degraus ósseos. Quanto a avaliação física, o perfil comportamental da criança pode interferir negativamente no diagnostico preliminar de lesões, sendo, em algumas vezes, necessário sedação ou anestesia geral para complementação da avaliação (Andrew et al., 2018).

As características como a flexibilidade e elasticidade óssea, mineralização reduzida do esqueleto facial, presença de estruturas gordurosas na face, suturas sem consolidação óssea e a menor pneumatização dos seios paranasais são fatores que contribuem para haver menor incidência de fraturas de face em pacientes pediátricos. Além disso, tais fatores anatômicos permitem o esqueleto facial absorver energia sem fraturas e caso ocorra a fratura possui maior probabilidade de que seja incompleta, configurando-se uma fratura de galho verde (Afrooz et al., 2012; Andrew et al., 2018).

Quanto aos agentes etiológicos mais comuns nesta população destacam-se em acidentes de trânsito, acidentes desportivos, quedas e agressões físicas (Chan et al., 2016; Gebran etal., 2019; Wang et al., 2018).

Em relação à localização predominante de fratura na face há divergências literárias, alguns autores acreditam que fraturas na região mandibular sejam mais frequentes (Chan et al., 2015; Ghosh et al., 2018; Liu et al., 2019), outros reportam que regiões como a orbitária, nasal e zigomática sejam mais frequentemente acometidas (Gebran et al., 2019; Le et al., 2020).

Sobre o tratamento de fraturas faciais em pacientes pediátricos, é essencial considerar as possibilidades de perturbação do crescimento ósseo (Mcggoldrick et al., 2019). Desta forma, uma abordagem não cirúrgica é indicada sempre que possível, porém o acompanhamento a longo prazo é recomendado para melhor avaliação da funcionalidade e estética (Andrew et al., 2018). Entretanto, fraturas deslocadas do corpo ou ângulo mandibular, fraturas do colo condilar com limitação mecânica de movimentos mandibulares, fraturas complexas e fraturas em áreas não dentais geralmente precisam de redução aberta e fixação interna (Eppely, 2005).

\section{Metodologia}

O presente estudo é um relato de caso, que consiste em um tipo de estudo descritivo, retrospectivo, qualitativo, realizado por meio da técnica de observação direta. A responsável legal pelo paciente consentio na divulgação dos dados e exibição das 
imagens por meio da assinatura do Termo de Consentimento Livre e Esclarecido e este caso foi conduzido respeitandoos princípios da Declaração de Helsinque (Pereira et al., 2018).

\section{Relato de Caso}

Paciente do gênero masculino, 1 ano e 10 meses, melanoderma, vítima de acidente automobilístico, foi encaminhado para a equipe de Cirurgia Geral do Hospital das Clinicas da Universidade Federal de Uberlândia para avaliação primária e solicitado avaliação secundária para o Serviço de Cirurgia e Traumatologia Buco-Maxilo-Facial. Durante anamnese, o responsável relatou que criança se encontrava no colo da mãe, durante a amamentação e ambos se encontravam sem cinto de segurança. Responsável negou perda de consciência após o trauma ou vômito, referiu que a criança apresentava alergia a amoxicilina, negou comorbidades, negou medicações em uso ou cirurgias pregressas.

Ao exame clínico inicial, o paciente apresentava edema significativo em região de face, presença de equimose em região bucal, equimose sublingual discreta, crepitação óssea mandibular durante manipulação, limitação de abertura bucal, dentição decídua presente com -má oclusão dentária e desnível oclusal. Diante dos achados clínicos, foram solicitados exames de imagem complementares, que incluíam radiografias P.A. (póstero-anterior) de face e laterais obliquas que evidenciaram fratura bilateral de mandíbula, incluindo parassínfise direita e ângulo esquerdo (Figura 1).

Figura 1: Radiografias pré-operatórias. A- P.A. de face; B- Lateral obliqua de mandíbula esquerda.

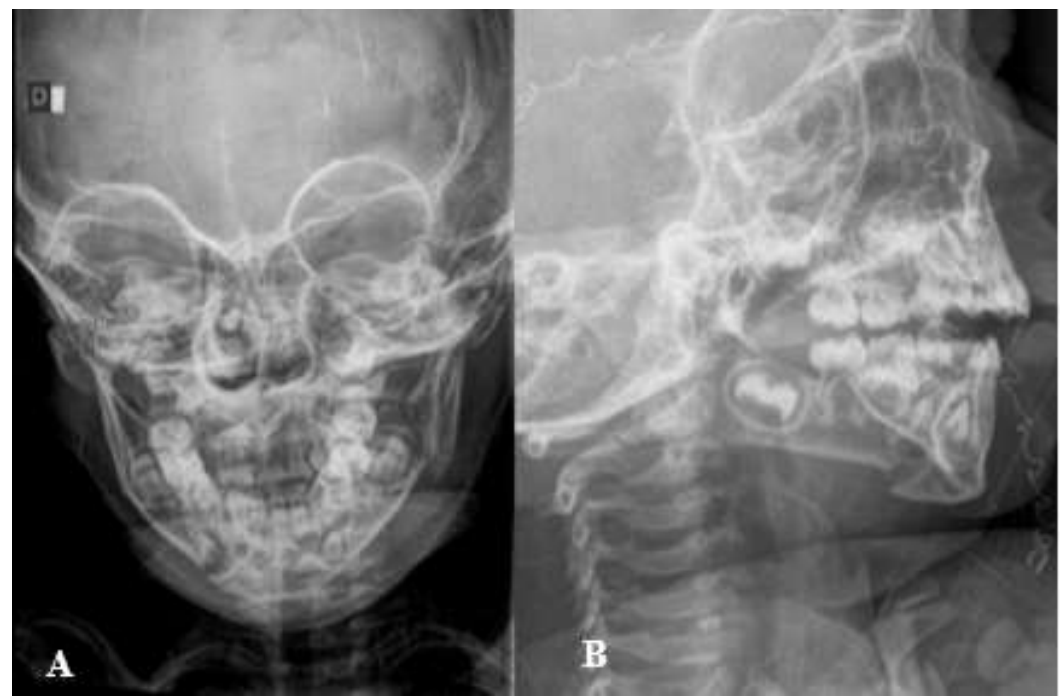

Fonte: Autores (2021).

Após discussão de caso com a equipe, considerando os aspectos anatômicos das fraturas, bem como seu deslocamento, optou-se pela modalidade de tratamento cirúrgico para realização de redução e fixação interna rígida de fratura bilateral de mandíbula.

$\mathrm{O}$ caso foi discutido com equipe de pediatria e anestesiologia que sugeriram intubação com uso de fibroscópio em virtude de significativo edema facial.

Paciente foi submetido a anestesia geral, com intubação nasotraqueal, com uso de fibroscópio infantil. $\mathrm{O}$ acesso à fratura de parassínfise mandibular foi conduzido por incisão em fundo de vestíbulo mandibular, dissecção de nervo mentual e descolamento mucoperiosteal. Foi realizado odontossíntese, utilizando dentição decídua presente. Após redução de fratura de parassinfise, a fixação interna rígida foi feita utilizando sistema 2.0, com parafusos bicorticais de $7 \mathrm{~mm}$ (Figuras 2 e 4). Em região de ângulo mandibular esquerdo, utilizou-se o acesso cirúrgico de Risdon, para abordagem da fratura de ângulo 
mandibular, que apresentava-se cominuta. Após redução da fratura, foi instalada de placa de fixação do sistema 2.0 em região de base de mandíbula, juntamente com parafusos bicorticais de $9 \mathrm{~mm}$. As suturas intra-orais foram realizadas com fio Vicryl® 3-0 (poliglactina multifilamentado) para reposicionamento de músculo mentual e sutura com fio monocryl®,4-0 (copolímero de poliglicaprona 25 monofilamentado) em mucosa intra-oral. As suturas em região de ângulo mandibular foram realizadas com fio Vicryl囚 3-0 (poliglactina multifilamentado) em planos e nylon 5-0 (monofilamentado) em pele (Figuras 02, 03, $04 \mathrm{e}$ $05)$.

Figura 2: Exposição de fratura de parassínfise mandibular.

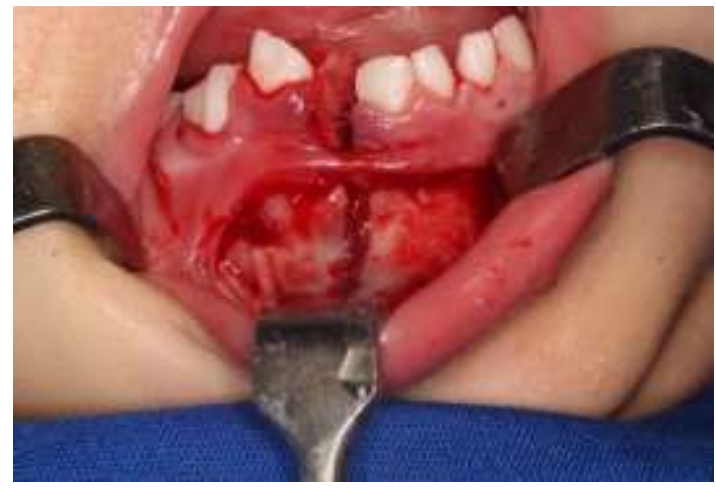

Fonte: Autores (2021).

Figura 4: redução de fratura e fixação interna rígida em região de parassínfise.

Mandibular.

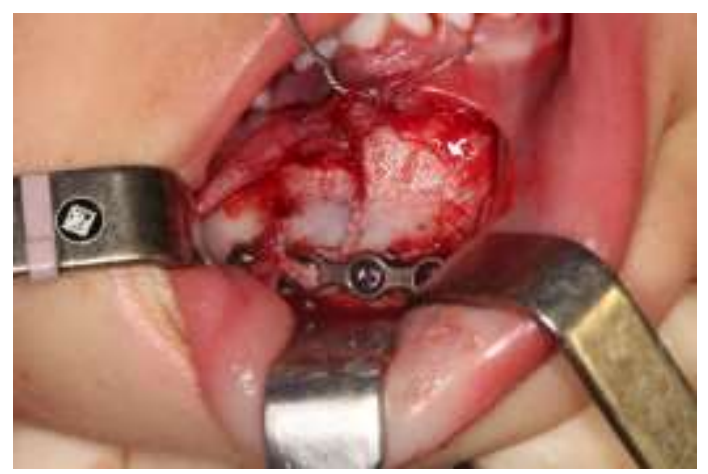

Figura 3: Exposição de fratura de ângulo mandibular.

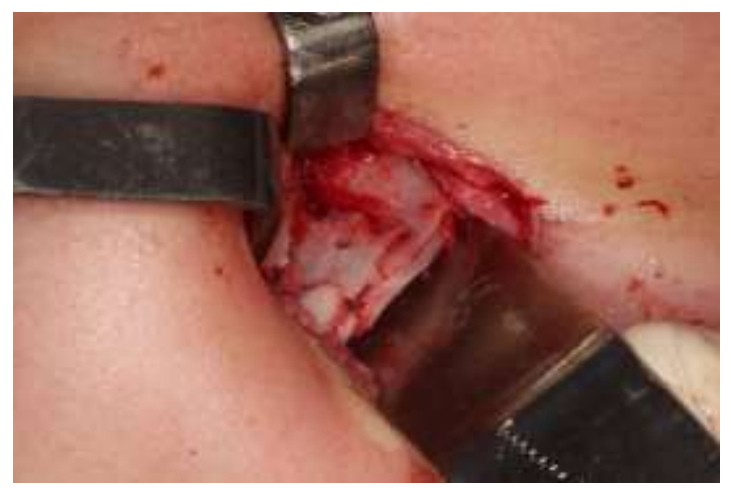

Fonte: Autores (2021).

Figura 5: redução de fratura e fixação interna rígida em região de ângulo mandibular.

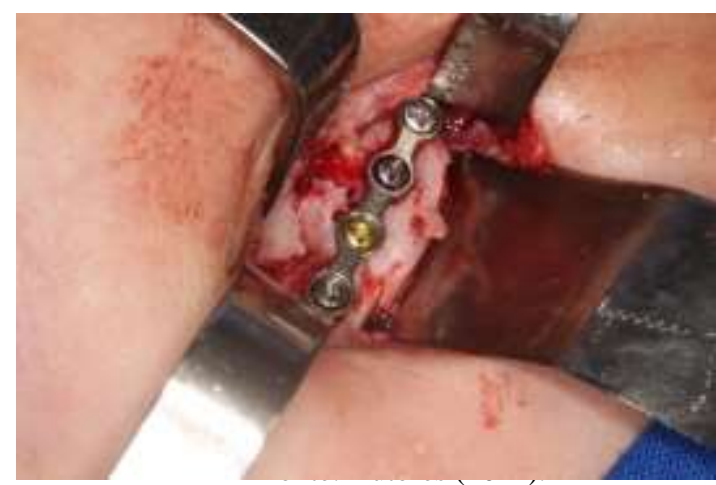

Como terapêutica medicamentosa durante o período de internação hospitalar, utilizou-se cefazolina, via endovenosa, $475 \mathrm{mg}$ de 6/6horas, dipirona 50mg/ml 6/6horas, morfina a critério médico. Em pós-operatório imediato paciente evoluiu com edema compatível com manipulação cirúrgica e ausência de sangramento ativo. Foi disponibilizado compressa de gelo intermitente, e orientado dieta liquida completa. Radiografias pós-operatórias evidenciaram boa redução de fraturas e material de osteossíntese em posição (Figura 06). 
Figura 6: Radiografias pós-operatória. A: P.A. de face; B: Lateral obliqua de mandíbula esquerda.

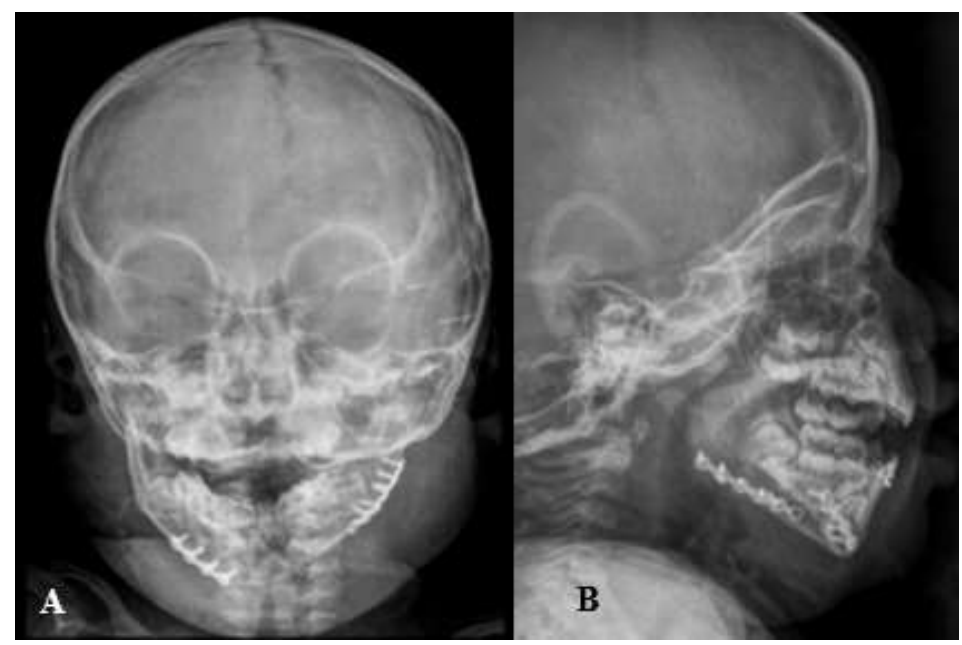

Fonte: Autores (2021).

Após 72 horas de pós-operatório, paciente recebeu alta hospitalar, em bom estado geral, com prescrição medicamentosa domiciliar de clindamicina $75 \mathrm{mg} / \mathrm{ml}, 2,5 \mathrm{mg}$ de 8/8hrs por 07 dias, ibuprofeno 50mg/ml, 13 gotas de $8 / 8 \mathrm{horas}$ por 03 dias, dipirona $50 \mathrm{~m} / \mathrm{ml}, 13$ gotas de 6/6horas por 03 dias.

Em acompanhamento de 7 dias de pós-operatório, paciente apresentou-se com ausência de deiscência de suturas extra-orais e intra-orais, higiene oral adequada, sendo realizada por responsável, ausência de sinais flogísticos e abertura bucal satisfatória.

Paciente foi acompanhado por período de 4 meses, ao fim do qual, não foi relatado nenhuma queixa pelo responsável, ou verificada anormalidade de cicatrização no paciente. Foram realizadas radiografias extra-orais para avaliação tardia, que demonstraram material de osteossíntese e cicatrização satisfatórios. Naquele momento, foi planejado remoção de material de osteossíntese. Sendo assim, paciente foi novamente submetido a procedimento cirúrgico sob anestesia geral, intubação nasotraqueal, onde foi realizado acesso intra-oral para remoção de material de osteossíntese de região de parassínfise mandibular e acesso extra-oral submandibular para remoção de material de osteossíntese da região de ângulo mandibular. (Figuras 7, 8, 9, 10).

Figura 7: Exposição de material de osteossíntese em parassínfise após 4 meses de ahordagem inicial.

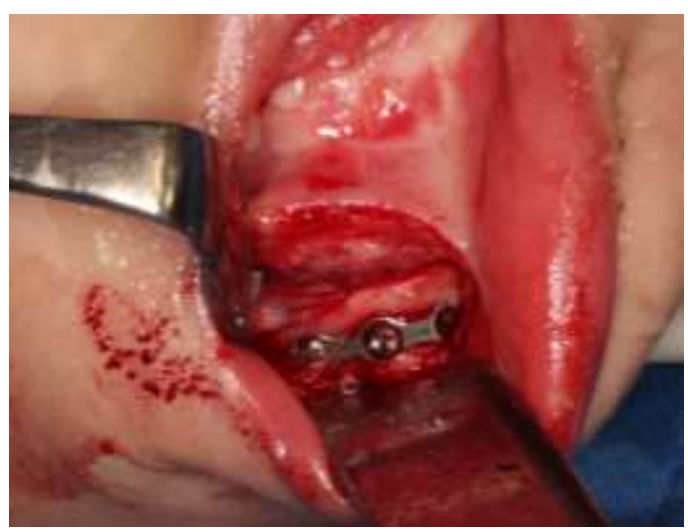

Fonte: Autores (2021).
Figura 8: Exposição de material de osteossíntese em ângulo após 4 meses de abordagem inicial.

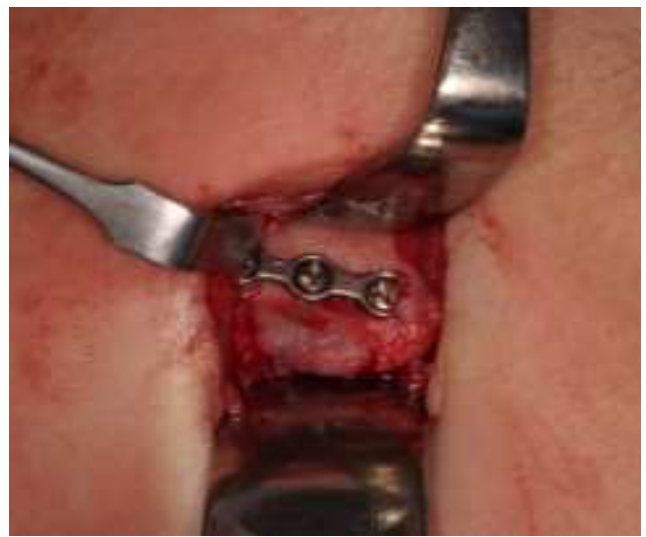

Fonte: Autores (2021). 
Figura 9: Aspecto clínico após a remoção do material de osteossíntese em parassinfise mandibular.

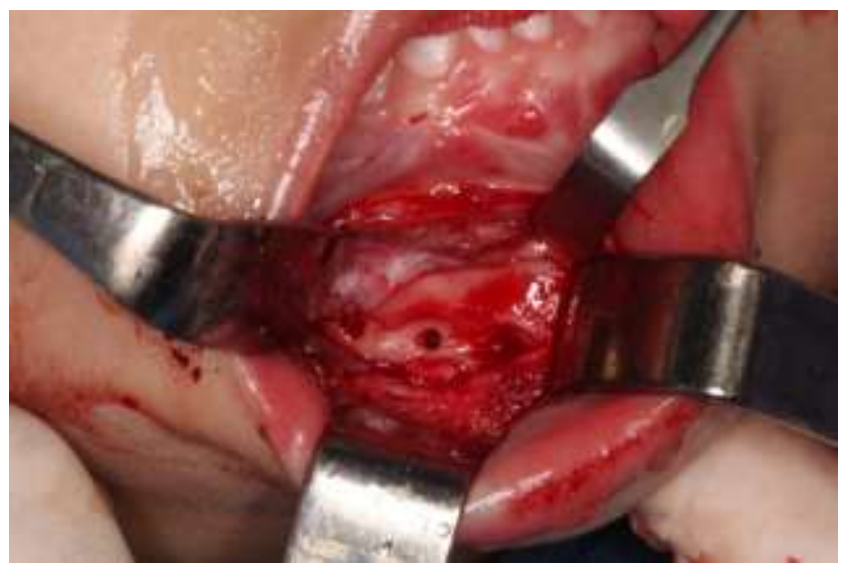

Fonte: Autores (2021).
Figura 10: Aspecto clínico após a remoção do material de osteossíntese em ângulo mandibular.

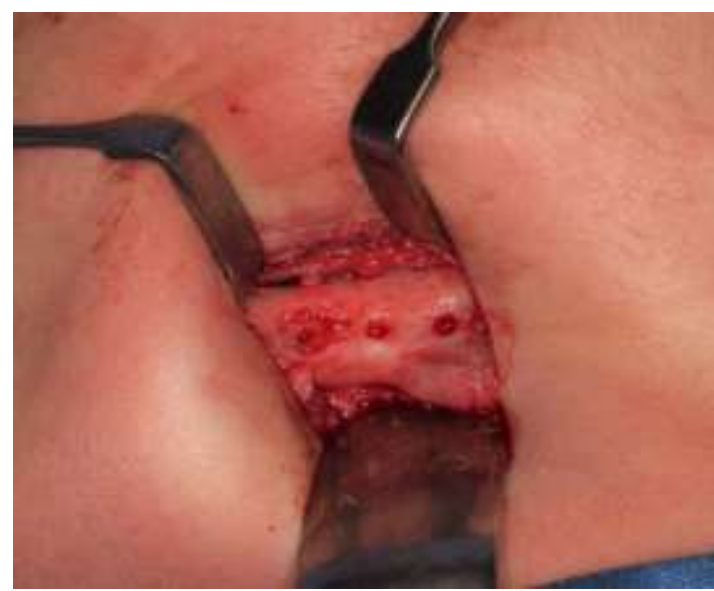

Fonte: Autores (2021).

Após 48 horas de pós-operatório, paciente recebeu alta hospitalar, em bom estado geral e em acompanhamento de 7 dias pós-operatório, paciente evoluiu com ausência de deiscência de suturas extra e intra-orais, higiene oral adequada sendo realizada por responsável, ausência de sinais flogísticos e abertura bucal satisfatória. Foram realizadas radiografias de controle, que evidenciaram consolidação de fraturas e presença de 01 parafuso em região de ângulo mandibular esquerdo (Figura 11). Optou-se por proservação quanto à permanência do parafuso, em virtude da ausência de sinais e sintomas associados.

Figura 11: Radiografia após remoção de material de osteossintese. A- P.A. de face; B- Lateral obliqua esquerda.

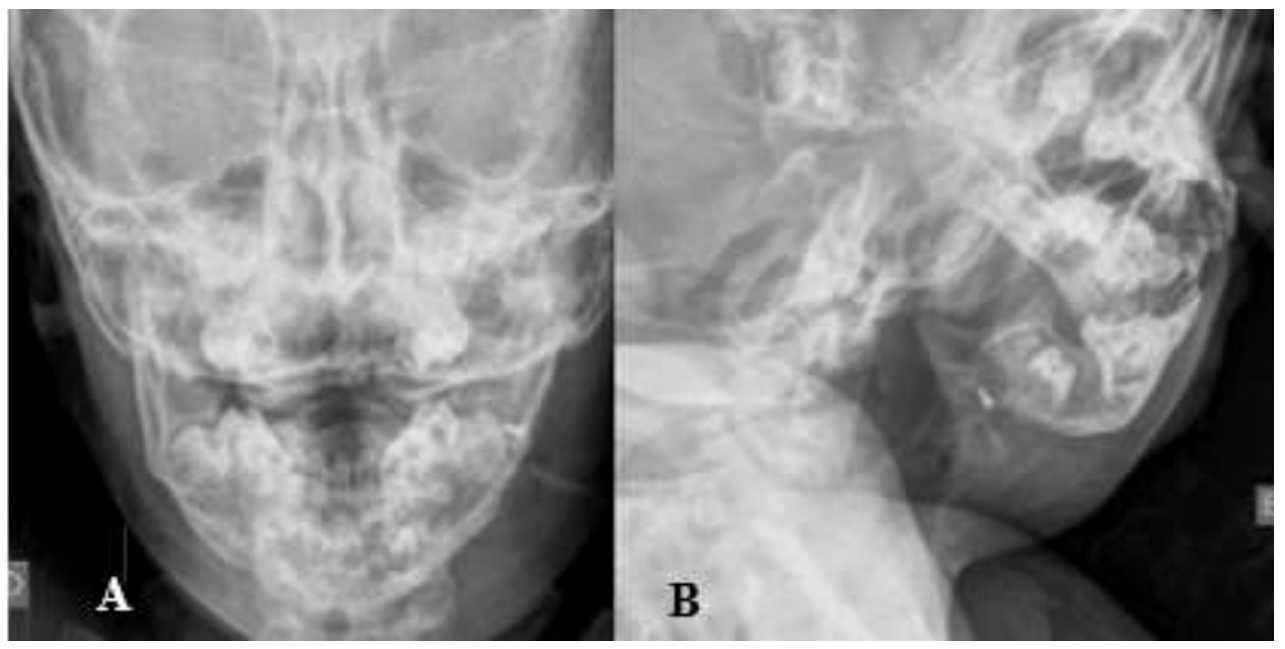

Fonte: Autores (2021).

Após abordagens cirúrgicas, paciente evoluiu com oclusão satisfatória, sem complicações pós-operatórias tardias, obtendo atualmente 1 ano de proservação e segue em acompanhamento anual com equipe de Cirurgia e Traumatologia BucoMaxilo-Facial do Hospital de Clínicas de Uberlândia até a finalização do processo de crescimento e desenvolvimento facial. (Figura 12). 
Figura 12: Oclusão após abordagens cirúrgicas. A- Vista lateral direita; B- Vista lateral esquerda; C- Vista frontal.

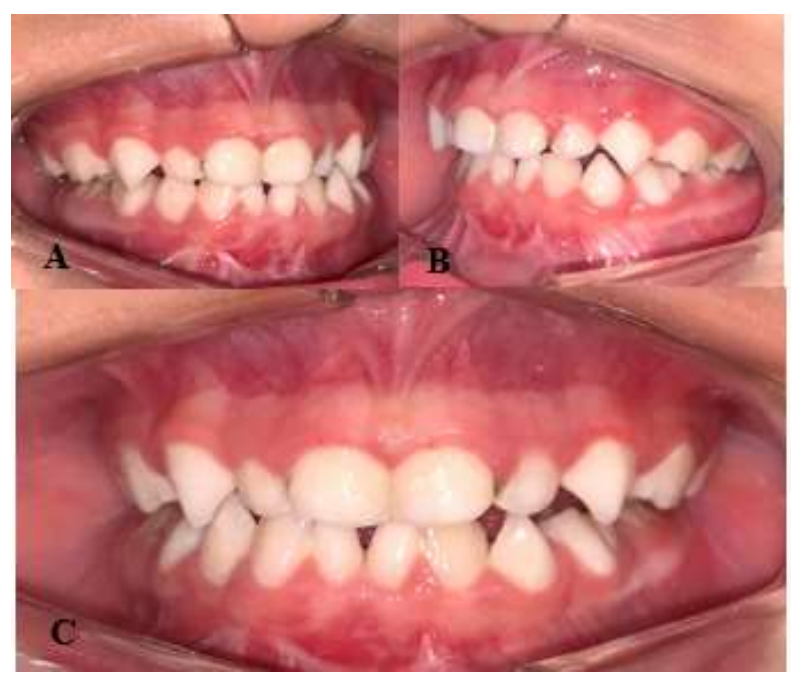

Fonte: Autores (2021).

\section{Discussão}

O esqueleto facial da criança sofre diversas alterações durante o crescimento, em especial quanto há traumas previos em zonas de crescimento, devendo cada caso ser avaliado individualmente, tendo em vista aspectos específicos da idade, como o alto potencial osteogênico (Pereira et al., 2021).

Apesar de haver discordância literária, a maioria dos estudos relata que a mandíbula é a região da face que mais sofre fraturas, seguido pela região orbital, nasal e maxilar (Afrooz et al., 2012; Chan et al., 2016; Ghosh et al., 2018; Liu et al., 2019). A distribuição anatômica das fraturas mandibulares varia com a idade: fratura condilar e fraturas subcondilares são mais comuns em pacientes jovens, no entanto, ambas diminuem com a idade, enquanto aumenta a incidência de fratura de corpo e de ângulo de mandíbula (Andrew et al., 2018).

O estágio de maturação do esqueleto facial pediátrico no momento da lesão tem um impacto significativo nos padrões de fratura facial e nas estratégias de tratamento. Uma revisão retrospectiva de prontuários foi realizada para todos os casos de fratura facial e observaram que pacientes com dentição mista foram significativamente mais propensos a ocorrência de fraturas do seio frontal e Le Fort, bem como lesões concomitantes graves com hemorragia intracraniana e traumatismo craniano (Berlin et al., 2021).

Uma das principais observações iniciais em casos de fraturas de mandíbula em pacientes pediátricos é a permeabilidade das vias aéreas. Sinais clínicos sugestivos de fratura de mandíbula incluem edema, equimose e lacerações, má oclusão dentária e trismo. Fraturas de mandíbula minimamente deslocadas podem ser tratadas através de repouso mandibular e imobilização, bem como com dieta líquida. Porém, deve-se levar em consideração que os principais objetivos no tratamento de fraturas de mandíbula em pacientes pediátricos são restauração da funcionalidade e proporcionar união óssea preservando o potencial de crescimento facial (Andrew et al., 2018).

As fraturas faciais pediátricas são tratadas de forma não cirúrgica em sua maioria, entretanto segundo Gefran et al., 2019, é possível verificar uma associação entre o aumento da idade e o tratamento operatório dessas fraturas, independente da demografia e de características da lesão. Além disso, com o avanço da idade, há uma tendência de aumento da gravidade da fratura e do grau de deslocamento necessitando de abordagem cirúrgica. Dados estes que se contrapoem ao caso em questão, onde foi possivel avaliar uma fratura grave com elevado grau de deslocamento em paciente de pouca idade. 
Em comparação com as fraturas faciais que ocorrem em adultos, fraturas faciais em crianças e adolescentes são relativamente incomuns. (Youssef et al., 2018; Imahara et al., 2008; Vyas et al., 2008) Pacientes com essas fraturas, muitas vezes requerem acompanhamento cuidadoso para futuras deformidades faciais secundárias a lesões do centro de crescimento, particularmente do septo nasal e côndilo mandibular e outros déficits de longo prazo (Bhardwaj e Kumar, 2015). Portanto, quando indicado o tratamento cirurgico de fraturas faciais pediatricas, este deve ser feito de maneira adequada e oportuna, a fim de evitar consequências no crescimento e desenvolvimento da criança (Bhardwaj \& Kumar, 2015; Youssef et al., 2018).

A fixação maxilomandibular em fraturas faciais pediátricas é bastante desafiadora, principalmente na dentição mista e os dispositivos intermaxilares tradicionais apresentam muitas limitações nesses pacientes. Atualmente, a utilização de botões ortodônticos para a fixação maxilomandibular transoperatória seguida do restabelecimento da oclusão, redução da fratura e fixação interna rígida pode ser um método que apresenta alta eficácia e rapidez no tratamento de fraturas em pacientes pediátricos (Vicente et al., 2020). No caso em questão, em virtude de não disponibilização de botões ortodônticos foram utilizados amarria dente a dente para redução de fratura e amarria ivy para a realização da correção maxilomandibular durante o transoperatório.

Quanto a remoção ou não do material de osteossíntese, não há um consenso na literatura em virtude de as publicaçoes mais recentes realizadas serem concentradas em relatos de caso e experiências institucionais individuais. Para Rudolf (2005), a remoção do material de osteossíntese em crianças é recomendado, mas não com a finalidade de prevenção de distúrbios do crescimento, e sim em virtude de sua movimentação com o crescimento da criança, podendo levar até mesmo ao deslocamento intracraniano. No caso em questão foi optado pela remoção do material de osteossintese na região mandibular bilateral após 4 meses do primeiro procedimento, sendo deixado apenas 01 parafuso, em virtude de estar coberto por tecido ósseo no momento da segunda abordagem cirúrgica.

Atualmente a fixação interna rígida é considera padrão ouro para o tratamento de fraturas de face, entretanto quando se trata de fraturas de face pediátricas podem ser utilizadas material de fixação reabsorvível, sem complicações envolvendo a consolidação tardia ou consolidação viciosa (Wong et al., 2017).

O uso de materiais de fixação reabsorvíveis nas fraturas faciais de pacientes pediátricos é bastante difundido, com boa aceitação e grandes vantagens sobre os materiais convencionais de titânio, principalmente pela eliminação de uma segunda cirurgia para retirada dos materiais (Pereira et al., 2021).Quanto às limitações na utilização da materiais reabsorvíveis estão a dificuldade de manuseio, dobramento da placa demanda muito tempo, além de ser mais complicada em relação ao titânio, os parafusos reabsorvíveis quebram facilmente durante a inserção e permitem uma compressão limitada, a esterelização deve ser realizada cuidadosamente por meio de irradiação ou com óxido de etileno; caso contrário, as propriedades mecânicas já limitadas serão reduzidas ainda mais e a reesterilização não é possível (Eppley, 2005; Rudolf, 2005).Por fim, pacientes pediátricos possuem vantagens regenerativas que incluem maior potencial osteogênico, taxa de cicatrização mais rápida, a dentição decídua ser temporária e capacidade de compensação dentária significativa. Provavelmente em virtude disso, a ocorrência de complicações como infecção, consolidação viciosa, não consolidação e má oclusão são relativamente raras em comparação a população adulta (Maqusi et al., 2012).

\section{Conclusão}

Fraturas faciais pediátricas, embora não muito comuns, necessitam de diagnósticos e tratamentos precisos e individualizados, visto que a conduta deverá causar a menor morbidade possível e devolver à criança estética e função adequadas sem prejudicar o crescimento ósseo fisiológico. O cirurgião bucomaxilo-facial deve manter o manejo do trauma facial infantil com alto índice de cuidado, realizar tratamentos com métodos adequados para cada idade e apresentar uma técnica cirúrgica muito precisa e pouco traumática, na busca de minimizar a ocorrência de complicações e iatrogenias 
Em virtude de não haver atualmente um consenso na literatura quanto ao tratamento e a remoção ou não do material de fixação interna e deste tipo de ocorrência não possuir uma prevalência alta, faz-se necessário o desenvolvimento de mais estudos relacionados ao tratamento deste tipo de fratura e o acompanhamento a longo prazo dos pacientes acometidos.

\section{Referências}

Afrooz, P. N., Grunwaldt, L. J., Zanoun, R. R., Grubbs, R. K., Saladino, R. A., Losee, J. E., \& Zuckerbraun, N. S. (2012). Pediatric facial fractures: occurrence of concussion and relation to fracture patterns. J craniofac surg. 23(5):1270-3. 10.1097/scs.0b013e31824e6447

Andrew. T. W., Morbia R., \& Peter Lorenz H.(2018). Pediatric Facial Trauma. Clin Plastic Surg. https://doi.org/10.1016/j.cps.2018.11.008

Berlin, R. S., Dalena M. M., Oleck N. C., Halsey J. N., Luthringer, M., Hoppe, C. I., Lee,E. S., \& Granick M. S. (2021). Facial Fractures and Mixed Dentition

- What Are the Implications of Dentition Status in Pediatric Facial Fracture Management?. J Craniofac Surg. Jan 7; 10.1097/SCS.0000000000007424

Bhardwaj, Y., \& Kumar, D. (2015). Pediatric maxillofacial trauma outcomes based on a survey of 65 patients: a prospective study of etiology, incidence and methods of treatment. J Maxillofac Oral Surg 14(3): 687e692.

Chan, K.H., Gao, D., Bronsert, M., Chevallier, K. M., \& Perkins, J. N. (2016). Pediatric facial fractures: Demographic determinants influencing clinical outcomes, Laryngoscope. 126(2):485-90.10.1002/lary.25457.

Imahara, S. D., Hopper, R. A., Wang, J., et al. (2008). Patterns and outcomes of pediatric facial fractures in the United States: a survey of the National Trauma Data Bank. J Am Coll Surg. 207:710-716

Gebran S. G., Wasicek,P. J., Elegbede, A., Ngaage, L. M., BChir, Liang, Y., Ottochian, M., Morrison, J. J., Rasko Y. M., Liang, F., Grant, M. P., \& Nam, A. J. (2019). Characterization of Age-Related Injury Patterns and Surgical Treatment of Pediatric Facial Fractures: Analysis of the National Trauma Data Bank. $J$ Craniofac Surg. 30(7) 10.1097/SCS.0000000000005789

Ghosh, R., Gopalkrishnnan, K., \& Anand, J. (2018). Pediatric Facial Fractures: A 10-year Study. J Maxillofac Oral Surg. 17(2):158-163. 10.1007/s12663-0160965-8

Eppley, B. L. (2005). Use of Resorbable Plates and Screws in Pediatric Facial Fractures. J Oral Maxillofac Surg .63:385-391. 10.1016/j.joms.2004.11.011.

Le, T.-M., Oleck, N. C., Liu, F.C., Halsey J. N., Hoppe, I. C., Lee, E. S., \& Granick M. S. (2020). Motor Vehicle Collision Injuries: An Analysis of Facial Fractures in the Urban Pediatric Population. J Craniofac Surg. 31(7):1910-1913. 10.1097/SCS.0000000000006671.

Liu, F. C., Le, T.-M., Oleck, N. C., Halsey, J. N., Hoppe, I. C., Lee, E., \& Granick, M. S. (2020). Pediatric Pedestrian Facial Fracture Patterns and Management Following Motor Vehicle Collisions. J Craniofac Surg. 31(1):265-268. 10.1097/SCS.0000000000006034.

Maqusi, S., Morris, D. E., Patel, P. K.,Dolezal, R. F., \& Cohen, M. N. (2012). Complications of pediatric facial fractures. J Craniofac Surg. 23 (4)1023-1027. 10.1097 / SCS.0b013e31824e7106

McGoldrick, D. M., Parmar, P., Williams, R., Monaghan, A., \& McMillan, K. (2019). Management of Pediatric Condyle Fractures J Craniofac Surg. 30(7)2045-2047. 10.1097/SCS.0000000000005787

Moffitt, J. K., Wainwright, D’A. J., Bartz-Kurycki, M., Wainwright, D. J., Demian, N., Teichgraeber, J. F., \& Greives, M. R. (2019). Factors Associated With Surgical Management for Pediatric Facial Fractures at a Level One Trauma Center. J Craniofac Surg. 30(3). 8540859. 10.1097/SCS.0000000000005245

Pereira A. S., Shitsuka D. M., Parreira F. J., \& Shitsuka R. (2018). Metodologia em pesquisa científica. UFSM - Santa Maria, RS. $1^{\circ}$ Ed.

Rodrigues, M. T. V., Schueng, F. E. A., Mendes, B. C. B. C., Vale, D. S., Souza, F. G., \& Nóia, C. F. C. F. (2020). A Simple Method of Transoperative Maxillomandibular Fixation in Pediatric Facial Fractures. J Craniofac Surg. 10.1097 / SCS.0000000000007279

Rudolf R.M. (2005). Treatment of Pediatric Facial Fractures: The Case for Metallic Fixation. J Oral Maxillofac Surg. 63:382-384. 10.1016/j.joms.2004.11.010

Santos, D. L. P., Miranda, M. L., Jacob, E. S., Guerra, R. C., Monnazzi, M. S., \& Vieira, E.H. (2021). Early Pediatric Multiple Facial Fractures Treatment With Resorbable Fixation. J Craniofac Surg. 10.1097/SCS.0000000000007382

Vyas, R. M., Dickinson, B. P., Wasson, K. L., et al. (2008).Pediatric facial fractures: current national incidence, distribution, and health care resource use. $J$ Craniofac Surg. 19:339-449

Youssef, P., Povolotskiy, R., Mukherjee T. J., Kandinov, A., \& Paskhover, B. Pediatric facial injuries: Hitting close to home, J J Craniofac Surg. 1539-1543. https://doi.org/10.1016/j.jcms.2018.05.054

Wong, F. K., Adams, S., Hudson, D. A. \& Ozaki, W. (2017).Use of Resorbable Fixation System in Pediatric Facial Fractures. J Craniofac Surg. 28 (3): 635637. 10.1097 / SCS.0000000000003413

Wang, H., Song, G., Ren, W., Zhou, Y., Li, C., Ou, L., Jun Liu, J., \& Xiang,L. Traumatic Facial Fractures in Children and Adolescents. J Craniofac Surg. 29(7) 10.1097/SCS.0000000000004964 